\title{
A CASE OF OLD HIP DISEASE
}

Shown at one of the Final F.R.C.S. classes.

The patient is a man aged 46 , complaining only of shortness of the left leg. No details are available of his previous medical history: apart from the value of the case as a demonstration of physical signs, its interest lies in attempting to diagnose the cause of the present condition.

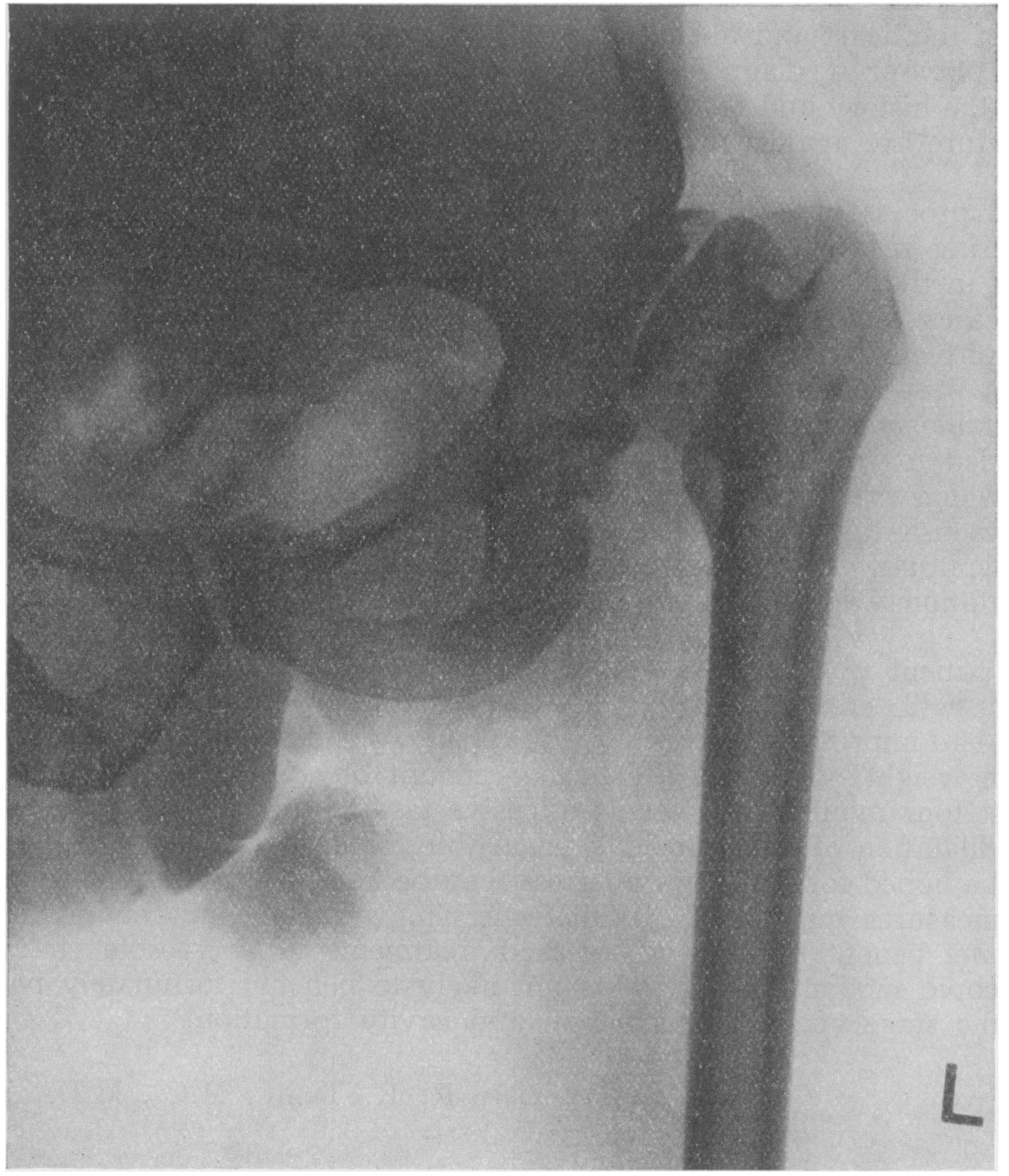

FIG. 1.

Radiogram showing condition of left hip joint. 
The story is that when he was aged one year, he had an acute illness, in the course of which he suffered from an abscess in the region of the left hip. This was incised: it is not known how long it took to heal, but he himself has no recollection of it being unhealed: the wound has never broken down, and at no time did he wear any form of splint. The leg has always been short.

The following are the physical signs:

The leg is two inches short. There is a scar one inch long situated on the inner side of the great trochanter. There is considerable wasting of all the muscles of the thigh, but there is no paralysis of any muscle. As a result of the muscular wasting the top of the great trochanter is unduly prominent, and owing to the almost complete disappearance of the neck of the femur, the great trochanter is very close to the anterior superior spine of the ilium. All movements of the hip joint are present but slightly limited, flexion being possible up to 90 degrees.

The radiogram is reproduced (Fig. I). The acetabulum is small and shallow : the head of the femur is distorted, but the joint line is clearly defined. There is almost complete disappearance of the neck. The left half of the pelvis is not so fully developed as the right. Immediately below the acetabulum there is some irregularity of the bone of which the significance is not clear.

The aetiology of the condition remains a matter for speculation. The possibilities considered were :-

r. Unreduced Congenital dislocation. $*$ The late results of this condition are usually stated to be unsatisfactory: if to an untreated congenital dislocation were added an acute infection, it is hard to conceive that the function of the joint and the appearance of the joint surfaces radiologically would be so good after 45 years.

2. Tuberculosis. It is hard to believe that a case of tuberculous infection treated so casually as was this case would lead to such a good functional result.

3. Acute infective arthritis. By exclusion this is the most reasonable diagnosis. Even so, there are difficulties to explain. A joint in which the infection has been so virulent as to lead to suppuration does not often retain such a full range of movement as is present in this hip. On the whole, it is true to say that function is better after a suppurative arthritis due to the streptococcus than in one following a staphylococcus. For the reasons given, a tentative diagnosis was made that the present condition of the hip is the result of an acute streptococcal arthritis. 